\title{
WiFi-based Location System for Indoor Assets Management
}

\author{
Siquan $\mathrm{Hu}^{1,2, a}$, Bowen $\mathrm{Li}^{1, \mathrm{~b}}$ and Chundong She ${ }^{3, \mathrm{c}}$ \\ ${ }^{1}$ School of Computer and Communication Engineering, University of Science and Technology \\ Beijing, Beijing, 100083, China \\ ${ }^{2}$ Chengdu Visual Microcosm Technology Ltd. Chengdu, 610072, Sichuan, China \\ ${ }^{3}$ School of Electronic Engineering, Beijing University of Posts and Telecommunications, Beijing, \\ 100876, China \\ ahusiquan@ustb.edu.cn, blbw_ustb@sina.com, cshurcd@vip.sina.com
}

\begin{abstract}
Keywords: WiFi; CC3200; Assets Location
Abstract. With the development of the wireless communication technology and intelligent wireless sensor, the positioning services based on WiFi are increasingly demanded. In this paper, we implement an indoor assets location system, based on WiFi and WiFi Received Signal Strength Indication (RSSI) measuring device which also includes a TCP transmission module. The system uses CC3200 for WiFi signal strength measurement and data transmission and python for the data processing in the cloud. We use the WiFi fingerprint calculated from RSSI which we get from a target device, by location-fingerprint database preset and special matching algorithms, we get the location of the target assets.
\end{abstract}

\section{Introduction}

With the technical advances in ubiquitous computing and wireless networking, there has been an increasing need to capture the context information (such as the location) and to figure it into applications [1]. Although the Global Positioning System (GPS) can provide accurate and reliable location information for the location service, it cannot be used effectively under indoor environment. To overcome this limitation, researchers have proposed many creative positioning technologies, such as sensor networks, RFID, and WiFi [2]. Among them, WiFi positioning technology has attracted wide attention for it is used widely in the world. As long as the deployment of a certain number of Access Point (AP) nodes, it can achieve precise positioning, therefore, it is feasible, practical to use WiFi networks and sensors to locate assets in the indoor environment.

WiFi-based indoor positioning can be divided into two categories according to the working basis, including those based on AP position approximation. Others include fingerprint based on the location. The former uses approximate location, location of target depending on the position of AP which has the strongest signal strength. The algorithm of fingerprint localization mainly consists of two parts, including offline establishment of location-fingerprint database and online positioning. Based on this, the authors design and implementation a WiFi-based indoor positioning system, which processing data in the cloud.

\section{Overall Design}

System Hardware. Hardware block of the system consists of two parts. One is the mobile facility consisting of an on-chip CC3200 with TCP transmission features, the other is data processing facility including a cloud server.

- Microcontroller. CC3200 is our main processor. It is a single-chip microcontroller unit (MCU) with built-in WiFi connectivity made by TI. It has an ARM Cortex-M4 core. There have 256 Kbytes of embedded SRAM including 12-bit ADCs, I2C, GPIO, UART and an embedded low-power WiFi module controlled by an independent network processor [3].

- Firmware Design. TI protocol stack provides built-in standard network protocols and built-in firmware to program the network processor.

Data Transmission. There are three steps for data transmission between the mobile facility and data processing server. Fig.1 shows the software flow chart of the system. 
- Collecting Data. First of all, the CC3200 use the WiFi module to measure the strength of the WiFi AP nearby and records the data in flash by the main processor. The main processor gets the data from network processor periodically.

- Establishing Connection. After data collection is complete, the CC3200 in the mobile facility initiates a TCP connection to the data processing server. In the connection establishment process, there is also a authentication mechanism between the server and the mobile facility to prevent receiving wrong data from an unknown device. We use a password which binds the device to validate the device.

- Transporting Data. The mobile facility writes its own device id and password to server to start the transport process. After authentication, the WiFi RSSI data will be transported to the server. The static function HTTPPostMethod will be used. When the server receives the data, the python functions will be used to process the data. Finally, the server shows the location of device on the web page.

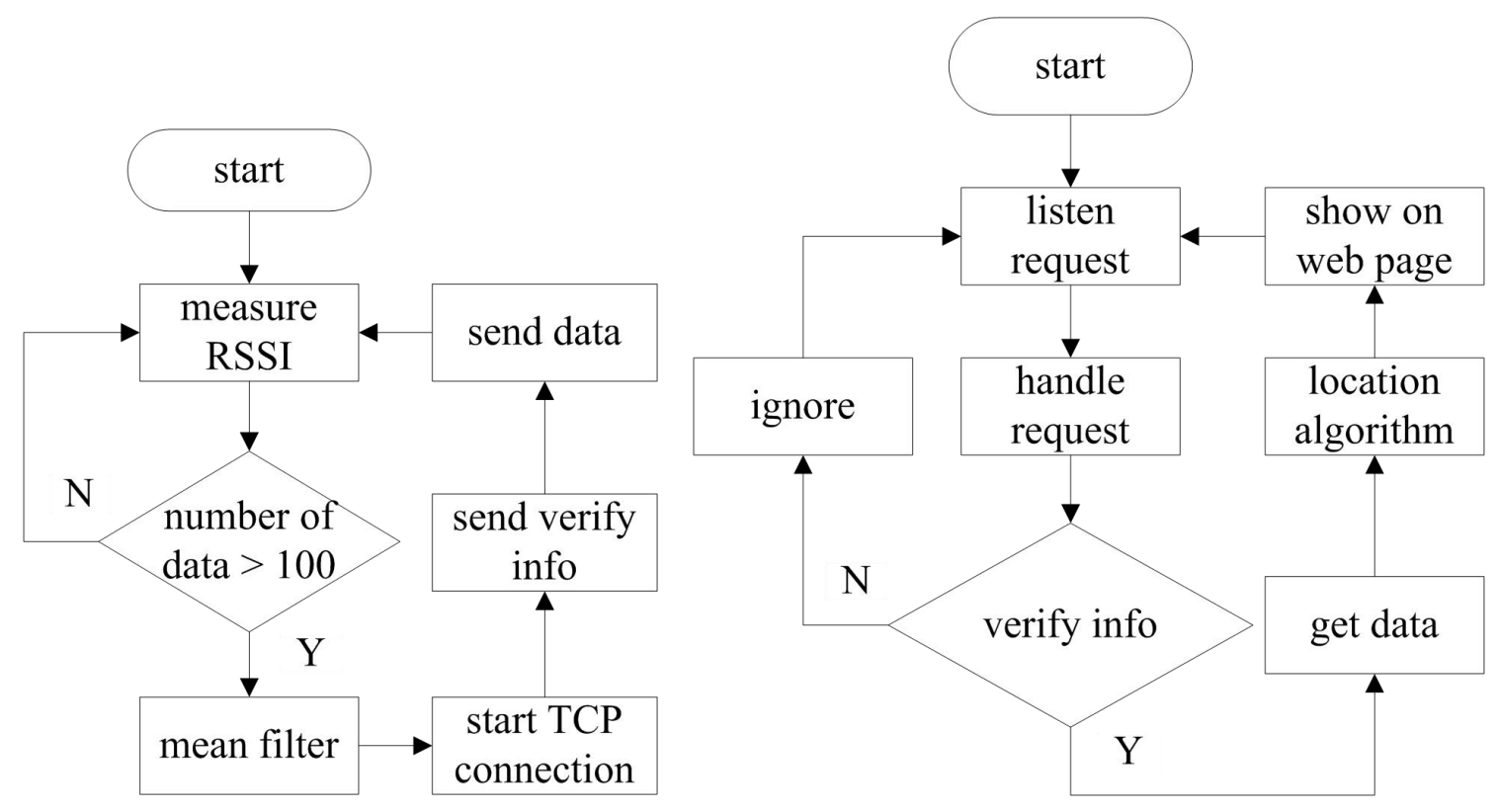

Fig.1 The software flow chart of mobile facility and server

\section{Algorithm Design}

In the data processing facility, we use python to process the data. Here we use and compare two typical methods to calculate the location of the mobile facility [4].

Trilateration method. This method gets the location of the device based on the basis of WiFi RSSI measurements and a signal propagation model inside the building. The propagation model can be obtained using simulations or with prior calibration measurements at certain locations. We can get the distance between AP and device according to the propagation loss model shown in Eq.1.

$$
P_{r}(d)=K-10 \varepsilon \lg (d)
$$

$P_{r}(d)$ is the resulted RSSI, $d$ is the distance, $\varepsilon$ is interference factor and $K$ is a constant which can be computed by equation shown in Eq.2.

$$
K=10 \lg \left[\frac{P_{r}\left(d_{0}\right)}{0.001 W}\right]+20 \lg \left(d_{0}\right)
$$

Calculated the distance, we can get the range of the device by equation set shown in Eq.3. 
Solve the equations shown in Eq.3, we can get intersection points of three circles of three AP. Finally, the coordinate of the device can be calculated by equation set shown in Eq.4.

$$
\begin{aligned}
& \left\{\begin{array}{l}
\left(X-X_{a}\right)^{2}-\left(Y-Y_{a}\right)^{2}=d_{1}{ }^{2} \\
\left(X-X_{b}\right)^{2}-\left(Y-Y_{b}\right)^{2}=d_{2}{ }^{2} \\
\left(X-X_{c}\right)^{2}-\left(Y-Y_{c}\right)^{2}=d_{3}{ }^{2}
\end{array}\right. \\
& \left\{\begin{array}{l}
\frac{\left(X_{a b}+X_{a c}+X_{b c}\right)}{3}=X_{0} \\
\frac{\left(Y_{a b}+Y_{a c}+Y_{b c}\right)}{3}=Y_{0}
\end{array}\right.
\end{aligned}
$$

Fingerprinting method. This method gets the WiFi RSSI in calibrated points and stored in offline database in advance. Then the location of the device can obtain by comparing with the RSSI values of calibrated points stored in database. Fig. 2 shows the algorithmic process.

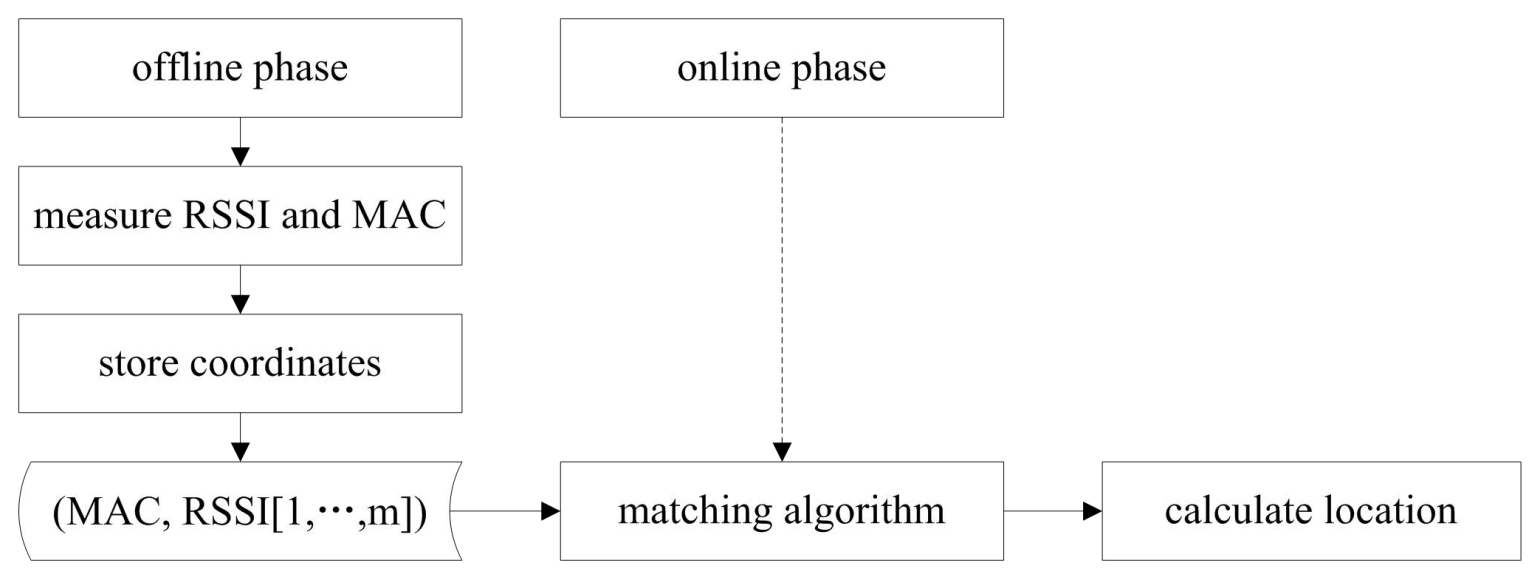

Fig. 2 The algorithmic process

At the offline phase, a certain amount of locations will be chosen, depending on the size and layout of the building. At each of these locations, a number of calibration measurements will be performed, all of the RSSI it can measure will be stored in database and others will be set to zero.

At the online phase, the server receives data from device. This information is compared with the values in database to obtain the location of the device. Matching the measurements and database according to equation shown in Eq.5.

$$
D_{m}=\left|\sqrt{\sum_{i=1}^{m}\left[R S S I_{D E V}(i)-R S S I_{D B}(i)\right]^{2}}\right|
$$

Here we set the RSSI which the device cannot measure to zero. Then pick up the coordinates corresponding to the smallest three $\mathrm{D}_{\mathrm{m}}$. The location of the device is the centroid of the three coordinates.

\section{Implementation and Test Results}

In order to get the best result, we have tested the two location algorithms in a building, Fig. 3 shows the AP distribution map in the test environment, the black spots represent the position of AP.

We choose three points in the building to test the trilateration method. For fingerprinting method, we measure the RSSI and MAC at each intersection of the grid in Fig.3 at the offline phase. At the online phase, we use the same three point as the trilateration method to test. The results of the 
testing showed below. Circles represent the position of AP, triangles represent the actual position and squares represent the calculated position. Fig. 4 shows the result of trilateration method and Fig. 5 shows the result of the fingerprinting method.

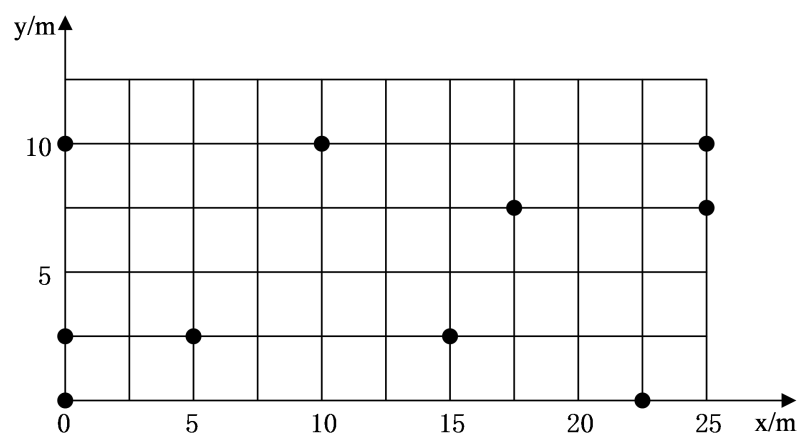

Fig.3 The AP distribution map

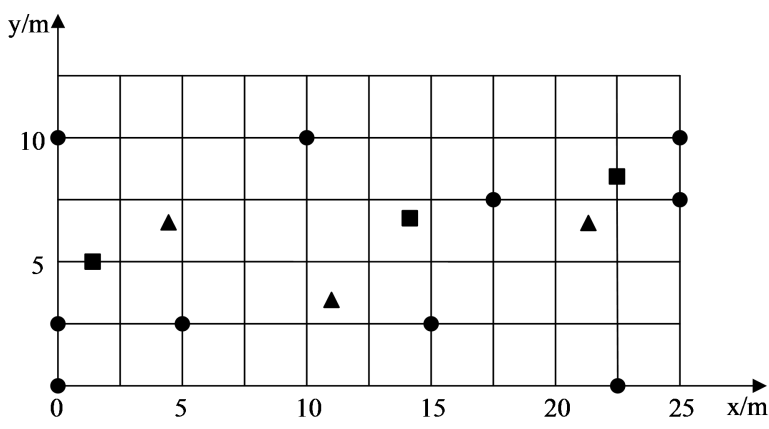

Fig.4 The result of trilateration method

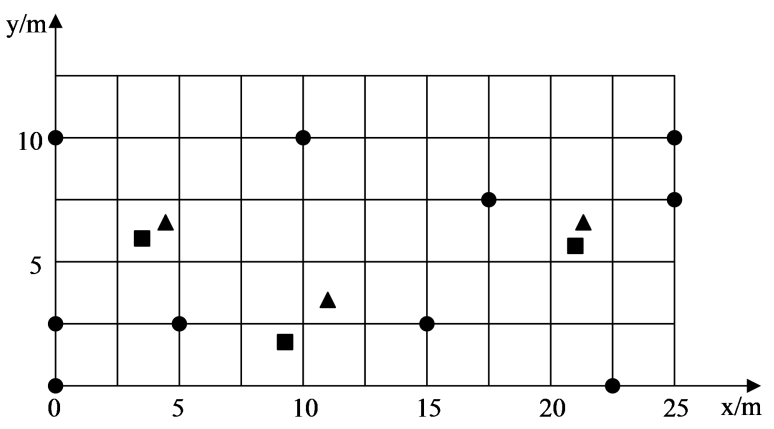

Fig.5 The result of fingerprinting method

According to the result of the test, the average error of the trilateration method is $3.261 \mathrm{~m}$ and the fingerprinting method is $1.594 \mathrm{~m}$. So we choose the fingerprinting method as the location algorithm in the system.

\section{Conclusions}

We have successfully designed and implemented the location system for indoor assets management, based on WiFi AP and mobile facility, including hardware device and software algorithm. Finally, it can give an accurate estimation without the knowledge of building structure. Therefore, the system can be applied in indoor environment as a useful assets location mechanism.

\section{Acknowledgements}

The study was supported by the National Natural Science Foundation of China (No. 91438120) and the Sichuan Science and Technology Support Program (No.2016GZ0339).

\section{References}

[1] H. Lim, L.C. Kung, J.C. Hou, H. Luo: Wireless Networks Vol. 16 (2010), p.405-420

[2] P. Jiang, Y.Z. Zhang, W.Y. Fu, H.Y. Liu, X.L. Su, J. Bajo: International Journal of Distributed Sensor Networks Vol. 2015 (2015)

[3] Information on http://www.ti.com/lit/ds/symlink/cc3200.pdf

[4] E.Mok, G.Retscher: Journal of Location Based Services Vol. 1 (2007), p.145-159

[5] Wes McKinney: Python for Data Analysis, (O'Reilly Media, California, 2012) 